\title{
1 Genomics analysis of hexanoic acid exposure in Drosophila species
}

3 Drum, Z.A. Lanno, S.M., Gregory, S.M., Shimshak, S.J., Ahamed, M., Barr, W., Bekele, B.,

4 Biester, A., Castro, C., Connolly, L., DelGaudio, N., Humphrey, W., Karimi, H., Karolczak, S.,

5 Lawrence, T., McCracken, A., Miller-Medzon, N., Murphy, L., Park, C., Park, S., Qiu, C., Serra,

6 K., Snyder, G., Strauss, A., Tang, S., Vyzas, C., and Joseph D. Coolon**.

7

8 'Department of Biology, Wesleyan University, Middletown, CT 06457

$11 *$ Corresponding Author:

12 Joseph D. Coolon

13 Wesleyan University

14123 Hall-Atwater

15 Middletown, CT 06459

$16860-685-2552$

17 Email: jcoolon@wesleyan.edu

18

19

20

21

22

Keywords: toxin resistance, host specialization, RNA-seq 


\section{$31 \quad \underline{\text { Abstract }}$}

32 Drosophila sechellia is a dietary specialist endemic to the Seychelles islands that has evolved to

33 consume the fruit of Morinda citrifolia. When ripe, the fruit of M. citrifolia contains octanoic

34 acid and hexanoic acid, two medium chain fatty acid volatiles that deter and are toxic to

35 generalist insects. $D$. sechellia has evolved resistance to these volatiles allowing it to feed almost

36 exclusively on this host plant. The genetic basis of octanoic acid resistance has been the focus of

37 multiple recent studies, but the mechanisms that govern hexanoic acid resistance in D. sechellia

38 remain unknown. To understand how $D$. sechellia has evolved to specialize on $M$. citrifolia fruit

39 and avoid the toxic effects of hexanoic acid, we exposed adult D. sechellia, D. melanogaster and

40 D. simulans to hexanoic acid and performed RNA sequencing comparing their transcriptional

41 responses to identify $D$. sechellia specific responses. Our analysis identified many more genes

42 responding transcriptionally to hexanoic acid in the susceptible generalist species than in the

43 specialist $D$. sechellia. Interrogation of the sets of differentially expressed genes showed that

44 generalists regulated the expression of many genes involved in metabolism and detoxification

45 whereas the specialist primarily downregulated genes involved in the innate immunity. Using

46 these data we have identified interesting candidate genes that may be critically important in

47 aspects of adaptation to their food source that contains high concentrations of HA.

48 Understanding how gene expression evolves during dietary specialization is crucial for our

49 understanding of how ecological communities are built and how evolution shapes trophic

50 interactions. 


\section{Introduction}

52 Insects have long been recognized as one of the most abundant and diverse groups of organisms

53 on the planet, with a large fraction of them feeding on plants (Jaenike et al. 1990, Stork 2018).

54 Many of these phytophagous insects have evolved to be highly host plant specific. The evolution

55 of such specialized interactions is often guided by specific plant chemistry, with most plants

56 responding to increased insect herbivory by the production of toxic secondary metabolites

57 (Jaenike et al. 1990, Petschenka and Agrawal 2016). This leads to an evolutionary arms race as

58 insects evolve resistance to these toxins (Heidel-Fischer and Vogel 2015). While host plant-

59 insect adaptions are well studied in the literature, less is known about the underlying genetic

60 mechanisms that contribute to the evolution of these complex ecological interactions (Ungerer et

61 al. 2007).

Drosophila sechellia feeds and oviposits primarily on Morinda citrifolia, a fruit highly

63 toxic to other Drosophila species (Legal et al. 1992). The plant produces ripe fruit year-round in

64 the Seychelles island archipelago, the sole location where D. sechellia are found (Legal et al.

65 1992) providing abundant and consistent resources. Upon exposure to the ripe fruit, other

66 Drosophila species display frantic behavior and wing movements, reduction in locomotor

67 activity, and death (Legal et al. 1994). Drosophila sechellia eggs are able to hatch and develop

68 on M. citrifolia fruit but the embryos of other Drosophila species die (Amlou et al. 1998). Unlike

69 its generalist sister species, D. sechellia prefer $M$. citrifolia to other hosts and are drawn to the

70 fruit from a long distance (R'Kha et al. 1991). Drosophila sechellia evolved resistance to the

71 toxins in $M$. citrifolia from an ancestral sensitive state (R'Kha et al. 1997) and this tolerance

72 provides $D$. sechellia a temporal advantage over other fruit fly species that can only lay eggs in

73 the fruit once it has rotten and the toxic volatiles are reduced. 
75 of the ripe fruit (Legal et al. 1994). This pulp is largely characterized by carboxylic acids,

76 primarily the fatty acids octanoic (OA) and hexanoic acid, (HA) which comprise 58\% and $19 \%$

77 of the volatile compounds found the ripe fruit respectively (Farine et al. 1996). While the genetic

78 basis of $D$. sechellia resistance to the most abundant and toxic compound in $M$. citrifolia fruit,

79 OA, has been characterized in previous studies (Dworkin and Jones 2009, Andrade Lopez et al. et al. 2019b), much less is known about the genes involved in HA resistance. In a study using

82 fatty acid concentrations equivalent to $1.5 \mathrm{~g}$ of ripe $M$. citrifolia fruit, OA treatment alone killed

83 all fruit fly species assayed except $D$. sechellia, while HA alone caused reversible knock-down in

84 other Drosophilids (Farine et al. 1996). In a more recent study using higher concentrations of

85 HA, mortality was observed in response to exposure to HA in D. melanogaster and D. simulans

86 and $D$. sechellia is significantly more resistant to HA induced mortality than sister species

87 (Peyser et al 2017, Lanno and Coolon 2019). Surprisingly, tests of the three major detoxification

88 gene families, cytochrome P450s (cyps), glutathione-S-transferases (GSTs) and esterases (Ests)

89 found that none was involved in derived HA resistance in D. sechellia suggesting an alternative

90 genetic mechanism must be involved in resistance. Studies have suggested that HA may be a

91 more efficient $D$. sechellia attractant than OA, while OA is a more potent repellant of generalist

92 species (Amlou et al. 1998). In a test using laboratory food medium supplemented with $0.5 \%$ of

93 either OA or HA, D. sechellia exhibited oviposition preference for media supplemented with HA

94 over OA (Amlou et al. 1998).

95 From a study analyzing the transcriptomic response of D. sechellia on OA, 104 genes

96 were found to be differentially expressed in response to OA (Lanno et al. 2017). This study 
97 showed that several Osiris genes, including Osi6 are upregulated in D. sechellia in response to

98 OA. Another study showed that RNAi mediated knockdown of Osi6 expression drastically

99 decreased survival in response to OA (Andrade Lopez et al 2017). Given that HA makes up

100 about of fifth of the volatile compounds found in M. citrifolia and produces unique effects on

101 behavior (Farine et al. 1996) and has an unknown and less common basis for toxin resistance

102 (Lanno and Coolon 2019, Peyser et al 2017), identifying the genes responding to HA is

103 necessary to understand how D. sechellia has specialized on M. citrifolia fruit, and may help

104 pinpoint genes that are involved in resistance to HA. By also analyzing the genes responding to

105 HA exposure in generalist Drosophila species D. melanogaster and D. simulans we can identify

106 derived gene expression responses specific to D. sechellia that may be critical for HA associated

107 traits (Coolon et al 2009). In this study, adult female D. sechellia, D. melanogaster, and D.

108 simulans flies were fed either control food or food supplemented with $0.23 \%$ HA and

109 significantly differentially expressed genes (DEGs) were identified using RNA-seq. Comparison

110 of the identified genes with those found to respond to OA (Lanno et al. 2017) and L-DOPA

111 (Lanno et al 2019), another highly abundant compound found in M. citrifolia fruit, identified

112 several genes common in response to OA, HA, and L-DOPA as well as genes unique to HA

113 suggesting these genes may play an important role in the evolved resistance and specialization of

114 D. sechellia to M. citrifolia.

\section{$116 \quad$ Methods}

\section{Fly strains and culture}


Drosophila sechellia (14021-0428.25), D. simulans (14021-0251.195), and D. melanogaster

119 (14021-0231.36) flies were reared on standard cornmeal medium under a 16:8 light:dark cycle

120 maintained at $20^{\circ} \mathrm{C}$.

121

RNA extraction, library preparation, and sequencing

123 Zero to three day post eclosion adult female flies were fed control food $(0.75 \mathrm{~g}$ Drosophila instant

124 medium Formula 4-24, Carolina Biological Supply Company) or identical food containing

$1250.23 \%$ hexanoic acid (HA). After 24 hours, three replicates of ten whole flies per species and per

126 treatment were homogenized and total RNA was extracted with a modified protocol of the

127 Promega SV extraction system (Coolon et al. 2013, Figure 1). RNA quality and quantity was

128 assessed using agarose gel electrophoresis and Nanodrop spectrophotometry. RNA was sent to

129 the University of Michigan Sequencing Core Facility for poly-A selection, cDNA synthesis, bar-

130 coded library preparation with TruSeq library preparation kits and sequencing on an Illumina

131 Hiseq 4000, generating 405,166,795 single-end $65 \mathrm{nt}$ sequencing reads for D. sechellia and 51nt

132 sequencing reads for D. melanogaster and D. simulans. (Table 1).

\section{BIOL310 Genomics Analysis}

135 The genomics analysis of RNA-seq data presented in this manuscript was performed by 20

136 undergraduate and 6 graduate students as part of a semester-long course at Wesleyan University

137 called Genomics Analysis (BIOL310). This is the third such manuscript (see Lanno et al. 2017

138 and Lanno et al. 2019a) made from this course where the aim is to provide undergraduate

139 students an early opportunity with a course-based research experience with active participation in

140 scientific discovery. Students in the course learn through engaging with never-before analyzed 
141 data using cutting edge genomics analysis techniques and bioinformatics tools through a

142 discovery-based independent study. Every student in the course contributed to the quality

143 control, analyses, write-up and interpretation of the findings, providing their own unique

144 perspective of the results and text written by each and every student was combined into this

145 manuscript with very little modification.

146 After sequencing output files were obtained from the University of Michigan Sequencing

147 Core (Table 1), fastq files containing raw sequencing reads were uploaded to the Galaxy

148 platform (Afgan et al. 2016) and an RNA-seq analysis pipeline was performed (Figure 1) as

149 previously described (Lanno et al. 2017 and Lanno et al. 2019a). Briefly, reads were assessed for

150 quality using FASTQC (Andrews 2010) and any overrepresented sequences were analyzed using

151 NCBI Blast (Altschul et al. 1990). Bowtie2 was used for mapping reads to the appropriate

152 reference genome for each species with default parameters (Langmead and Salzberg 2012), with

153 the most recent genomes for each species available at the time of analysis acquired from

154 Ensembl (www.ensembl.org, Yates et al. 2016) (D. sechellia:

155 Drosophila sechellia.dsec caf1.dna.toplevel.fa, D. simulans:

156 Drosophila_simulans.ASM75419v3.dna.toplevel.fa and D. melanogaster:

157 Drosophila melanogaster.BDGP6.dna.toplevel.fa). The Bowtie2 output files were analyzed

158 using Cuffdiff (Trapnell et al. 2010), which performs gene expression quantification and

159 differential gene expression analysis using the aforementioned genome file along with the most

160 recent annotated .gff3 file for each genome available at the time of analysis acquired from

161 Ensembl (D. sechellia: Drosophila_sechellia.dsec_caf1.42.gff3, D. simulans:

162 Drosophila_simulans.ASM75419v3.42.gff3 and D. melanogaster:

163 Drosophila_melanogaster.BDGP6.95.gff3). In Cuffdiff, geometric normalization and library size 
correction was performed, along with bias correction using the reference genome, giving an

165

166

167

168

169

170

171

172

173

174

175

176

177

178

179

180

181

182

183

184

185

186

output of DEGs for each species following false discovery rate multiple testing correction

(Benjamini \& Hochberg 1995, q < 0.05). Data was visualized using R (R Core Team, 2013). In

order to compare gene expression results across species, we obtained all 1:1:1 orthologs from $D$.

sechellia, D. simulans and D. melanogaster from Flybase (Thurmond et al. 2019). DEGs

following $D$. sechellia exposure to OA or L-DOPA were downloaded from online databases

(Lanno et al. 2017, Lanno et al. 2019a). GO term enrichment was performed on D. melanogaster

orthologs for each species using GeneOntology.org (www.geneontology.org, Ashburner et al.

2000, Carbon et al.2021, Mi et al.2019). KEGG pathway analysis was performed using the $D$.

melanogaster ortholog for each DEG from each species

(https://www.kegg.jp/kegg/tool/map pathway1.html, Kanehisa and Sato 2020).

Data accessibility

All RNA-seq data generated in this manuscript have been submitted to the NCBI Gene

Expression Omnibus under accession number XXXXX (to be available at time of publishing).

Supplemental Tables for this manuscript have been uploaded to GSA figshare.

\section{$\underline{\text { Results }}$}

\section{Differential gene expression in response to $\mathrm{HA}$ treatment}

In order to identify candidate genes that are important in D. sechellia host specialization and

evolved resistance to HA we sought genes that have altered expression levels in response to HA

exposure. Previous studies have shown that such environmentally plastic gene regulation can

indicate importance of that gene's function in that environment making identified genes good 
187

188

189

190

191

192

193

194

195

196

197

198

199

200

201

202

203

204

205

206

207

208

209

candidates for D. sechellia HA associated traits (Coolon et al 2009, Lanno et al 2017, Lanno et al 2019). To quantify gene expression response to HA we performed RNA-sequencing (RNA-seq) on adult female flies after exposure to control food and compared this to flies fed food containing $0.23 \%$ HA. Because many of the transcriptional responses to HA might be non-specific, we measured gene expression responses in D. sechellia, D. melanogaster, and D. simulans to identify those responses (or loss of response) that are restricted to D. sechellia representing changes that might contribute to its unique phenotypes.

Using this approach we identified 841 genes differentially expressed by $D$. melanogaster (Figure 2A,D; Table S1), 743 genes were differentially expressed by D. simulans (Figure 2B,E; Table S2) and only 93 genes were differentially expressed in D. sechellia (Figure 2 C,F; Table S3) in response to HA. No significant difference in the number of upregulated genes (50/93) vs downregulated genes (43/93) was observed in D. sechellia in response to HA (Binomial Exact Test, $\mathrm{p}=0.1066$ ). In $D$. simulans, there was a significant difference in the number of upregulated genes (69/743) compared to the number of downregulated genes (674/743) in response to HA (Binomial Exact Test, $\mathrm{p}=2.2 \mathrm{e}-16$, Figure 2B,E). In $D$. melanogaster, there was also a significant difference in the number of upregulated genes (171/841) compared to the number of downregulated genes $(670 / 841)$ in response to HA (Binomial Exact Test, $\mathrm{p}=2.2 \mathrm{e}-16$, Figure 2A,D). In D. sechellia, there were 39 DEGs identified that responded to HA that do not have annotated D. melanogaster orthologs 27 of these genes were 5.8S rRNAs, two snoRNAs, and 7 genes of unknown function. Of the $275.8 \mathrm{~S}$ rRNAs, all 27 were upregulated (Binomial Exact Test, $\mathrm{p}=7.451 \mathrm{e}-09$ ). In D. simulans, of the 743 DEGs there were annotated D. melanogaster orthologs for 673 genes. For the remainder of the analysis, only genes with known D. melanogaster orthologs are considered to allow functional 
210

211

212

213

214

215

216

217

218

219

220

221

222

223

224

225

226

227

228

229

230

231

232

interpretations of DEGs, and for all subsequent analyses the D. melanogaster ortholog name was used. This filtering resulted in 673 differentially genes in D. simulans, 841 in D. melanogaster and 54 in D. sechellia used in subsequent analyses.

\section{Identifying functional enrichment in DEGs}

To identify the biological pathways that are involved in responses to HA in D. sechellia, D. melanogaster, and D. simulans KEGG analyses of the upregulated and downregulated genes in each species were performed. These analyses show that many different metabolic and detoxification pathways along with proteins that localize to the lysosome are changing in response to HA exposure in D. melanogaster and D. simulans, whereas very few genes in these pathways are responding in D. sechellia (Figures 4A-B). In D. sechellia, many of the of the genes downregulated in response to HA are involved in Toll and Imd immune signaling whereas this was much less prominent in D. melanogaster and D. simulans DEGs (Figure 4C).

To understand which biological and cellular processes are being altered in D. sechellia, D. melanogaster, and D. simulans in response to HA, Gene Ontology (GO) term enrichment analysis was performed (Table S16-S18). In D. sechellia, GO term enrichment analysis of DEGs

for cellular component showed a significant enrichment for extracellular region genes $(\mathrm{p}=4.83 \mathrm{e}-$ 05), suggesting that several of the genes responding to HA exposure have proteins that are secreted. For biological process GO term enrichment analysis, processes involved in the antibacterial humoral response were significantly enriched $(\mathrm{p}=1.75 \mathrm{e}-08)$. In $D$. sechellia, no molecular function processes were significantly enriched. In D. melanogaster, there was also significant enrichment for GO terms for extracellular region genes $(p=4.42 e-04)$, along with genes found inside the nucleolus $(\mathrm{p}=8.40 \mathrm{e}-04)$. For biological process, GO terms associated 
233 with genes involved in the antibacterial humoral response were significantly enriched as in $D$.

234 sechellia $(\mathrm{p}=3.02 \mathrm{e}-02)$, as were other processes involved in the Drosophila immune response.

235 The D. melanogaster HA response also was enriched for genes involved in ribosome biogenesis

236 ( $\mathrm{p}=3.66 \mathrm{e}-02)$. In D. simulans, genes found inside the nucleolus were significantly enriched in

237 the set of HA responsive genes $(\mathrm{p}=2.65 \mathrm{e}-02)$. In an analysis of DEGs in D. simulans that are

238 upregulated, genes involved in Notch signaling were significantly enriched $(\mathrm{p}=4.11 \mathrm{e}-04)$

239 alongside genes involved in vitelline membrane and chorion formation $(\mathrm{p}=2.56 \mathrm{e}-02)$. In $D$.

240 sechellia, upregulated genes were significantly enriched for the larval serum protein complex (p

$241=1.96 \mathrm{e}-02)$. Downregulated genes were significantly enriched with antibacterial humoral

242 response GO terms $(\mathrm{p}=3.24 \mathrm{e}-10)$ along with the response to hyperoxia $(\mathrm{p}=1.52 \mathrm{e}-03)$. These

243 downregulated genes were also enriched for the extracellular region $(\mathrm{p}=4.17 \mathrm{e}-05)$. In $D$.

244 melanogaster, upregulated genes were significantly enriched to be involved in the larval serum

245 protein complex $(\mathrm{p}=1.23 \mathrm{e}-03)$ and were enriched intracellularly $(\mathrm{p}=4.02 \mathrm{e}-16)$ and within

246 intracellular organelles (1.32e-13). These upregulated genes were significantly enriched in many

247 biological processes, including chromatin silencing $(\mathrm{p}=3.25 \mathrm{e}-03)$, ecdysone receptor-mediated

248 signaling $(\mathrm{p}=1.89 \mathrm{e}-02)$, and chorion assembly $(\mathrm{p}=2.25 \mathrm{e}-02)$. In D. melanogaster,

249 downregulated genes were significantly enriched for the antibacterial humoral response $(\mathrm{p}=$

$2504.52 \mathrm{e}-03)$ and for the defense response to Gram-positive bacteria $(p=5.07 \mathrm{e}-04)$. These

251 downregulated genes were significantly enriched to be localized to the extracellular region $(\mathrm{p}=$

$252 \quad 1.25 \mathrm{e}-04)$.

254 Comparing DEGs identified in response to HA in D. melanogaster, D. simulans, and D. 
In order to identify genes with $D$. sechellia specific responses to HA, we compared the

257 DEGs from the three species when exposed to HA (Figure 3A). We identified 32 genes that

were differentially expressed by $D$. sechellia flies exposed to HA that were not responsive to HA

259 exposure in both D. melanogaster and D. simulans flies (Figure 3A, Table S13). In order to

identify those genes where $D$. sechellia specific loss of response to HA was observed we selected

those genes with significant changes in response to HA in both D. melanogaster and D. simulans

262 that were not significantly differentially expressed by D. sechellia in response to HA. This

263 analysis yielding a total of 213 genes with this expression pattern in our data (Table S10).

264 Interestingly, only 2 genes were identified, $C G 13114$ and $F b p 1$, that had significant response to

265 HA in all three species, with the expression of both increasing in $D$. simulans and decreasing in

266 D. sechellia and D. melanogaster (Figure 3A, Table S9).

Common transcriptional responses of $D$. sechellia exposed to $H A, O A$ and L-DOPA suggests

270 To assess the overlap in transcriptional responses of D. sechellia to both OA and HA, the

271 predominant fatty acid volatiles in M. citrifolia, along with 3,4-dihydroxyphenylalanine (L-

272 DOPA), which is found in $M$. citrifolia fruit and is important for the specialization of $D$.

273 sechellia to this fruit (Lanno et al. 2019a, Lavista-Llanos et al. 2014), we compared DEGs

274 between our HA treatment, DEGs identified in a previous study using a $0.7 \%$ OA treatment

275 (Lanno et al. 2017), and DEGs identified in a previous study of responses to $10 \mathrm{mg} / \mathrm{mL}$ of L-

276 DOPA added to the fly food (Lanno et al. 2019a). Treatment with OA treatment yielded 103 D.

277 sechellia genes with $D$. melanogaster orthologs that were significantly differentially expressed.

278 Treatment with L-DOPA yielded 643 D. sechellia genes with D. melanogaster orthologs that 
were significantly differentially expressed. Comparison of genes responsive to HA, OA, and L-

(Figure 3B, Table S6). Interestingly, of the 19 shared DEGs with D. melanogaster orthologs

291 Interestingly, in response to OA and HA, D. sechellia downregulated genes involved the humoral

292 immune response (AttC, CecA2, Def, DptB, Dro, edin, and PGRP-SB1 Table S4).

\section{$\underline{\text { Discussion }}$}

295 Understanding the genetic basis of how organisms evolve to occupy different ecological niches

296 and adapt to their environments is crucial to understanding the evolution of plant and animal

297 interactions. Insect-host plant specialization is an excellent example of the evolution of such

298 interactions and has been the subject of numerous ecological studies. While the phenomenon is

299 well documented, the genetic basis of evolved host specialization is still not widely understood.

300 Here we focus on the specialization of $D$. sechellia to feed almost exclusively on one host plant,

301 M. citrifolia because it is an excellent model to understand the genetic basis of dietary 
specialization. This is in part because it is has evolved recently and very closely related to the genetic model generalist species D. melanogaster. Fortuitously, we can take advantage of the wealth of genetic tools and information about D. melanogaster and sister species to understand the evolution of dietary specialization in this group (Groen and Whiteman 2016). 2009, Matsuo et al. 2007). The mechanisms that drive this attraction may be through changes in gene expression and may be reflected in these predicted regulatory networks. Drosophila

316 sechellia downregulates many genes involved in the Imd and Toll immune pathways when 317 exposed to both HA and OA, suggesting that somehow these two medium chain fatty acids are

318 interacting with negative effectors of Imd signaling. As these two immune pathways have similar 319 and overlapping target genes, further analysis is needed to determine if both pathways are 320 involved in these interactions (Hanson and Lemaitre 2020). This interaction between D. sechellia 321 and the volatiles from $M$. citrifolia reduces the immune humoral response, which could cause

322 alterations in gut microbiota composition that aids in the detoxification of relevant plant

323 secondary metabolites. E(spl)mgamma-HLH is a Notch responsive Myc-like transcription factor

324 that has been shown to interact with Relish, the main regulator of Imd immune signaling 
325 (Dushay, Åsling, and Hultmark 1996) through recent yeast two-hybrid assays (Shokri et al. 2019) and is upregulated in D. sechellia in response to both OA and HA exposure (3B Table S4). The plastic response of insect immune systems allows them to fend off pathogens when needed, but also allow for the management of endosymbionts (Vilcinskas 2013, Login et al. 2011). Unlike its generalist sister species, Drosophila sechellia was previously shown to lack an immune response when confronted with parasitic wasps, hinting that immune system responses in this species may be unlike its generalist sister species (Salazar-Jaramillo et al. 2017). Additionally, prior work showed that free fatty acids are involved in regulating immune responses in mammals (Alvarez-Curto and Milligan 2016), and hexanoic acid priming of plants can activate the jasmonic acid pathway to increase plant resistance to fungal pathogens (Aranega-Bou et al. 2014; García-Robles et al. 2013). Further study comparing the microbiomes between $D$. sechellia and D. simulans and how they may change from feeding on M. citrifolia fruit as well as how each responds to pathogen challenge in this context would help to understand why $D$. sechellia is downregulating its immune response when there could be a serious fitness cost of this action. Recent studies have shown that D. melanogaster uses sweet tasting gustatory receptor neurons to sense OA and HA (Masek and Keene 2013; Tauber et al. 2017; Chen and Amrein 2017). Our data shows that upon exposure to HA, only D. sechellia significantly upregulates

343 another odorant binding protein, Obp56a. In contrast to HA treatment, in response to OA $D$. 344 sechellia adults do not change expression of any odorant binding proteins (Lanno et al. 2017).

345 Both $D$. simulans and $D$. melanogaster both downregulate the expression of Opb57a in response

346 to HA (Table S10). Recently, studies examining the gustatory and behavioral basis of the

347 attraction of $D$. sechellia to noni have shown that Or22a neurons in the fly brain are involved in 
the attraction to noni (Auer et al. 2020), as are $\mathrm{Or} 85 \mathrm{~b} / \mathrm{c}$ and $\mathrm{Ir} 75 \mathrm{~b}$ neurons (Prieto-Godino et al. 2017). Orco mutant D. sechellia flies lose olfactory responses to both 2-heptanone and 1hexanol, two compounds found in ripe noni fruit (Auer et al. 2020). Further work on the role of these genes in D. sechellia and the regulatory mechanisms responsible for the change of odorant binding protein expression in response to HA may help to elucidate how D. sechellia has evolved to specialize on $M$. citrifolia in the generalist species D. melanogaster and D. simulans (Tables S1-3). Drosophila sechellia appears to be downregulating many genes involved in the humoral immune response whereas $D$. melanogaster and $D$. simulans alter the expression of genes involved in many different metabolic pathways (Figures 4A-4C). As generalist insects feed on many different plants which produce different secondary metabolites to defend themselves from predators, perhaps generalist species have a more plastic regulatory response to subvert toxicity whereas the specialist $D$. sechellia is resistant to the toxicity of its host through a specific constitutive mechanism. A recent study comparing the fitness of D. melanogaster to D. sechellia larvae fed different food sources showed a loss of carbohydrate metabolic responses in D. sechellia, as they have specialized on a

364 fruit with a relatively low sugar content, M. citrifolia (Watanabe et al. 2019). Of the many 365 significant genes differentially expressed in D. melanogaster and D. simulans, most DEGs are not drastically differentially expressed in HA treatment compared to controls (Figures 2D and 2E). Drosophila sechellia conversely alters the expression of relatively far fewer genes, but many of the DEGs in response to HA are drastically differentially expressed (Figure $\mathbf{2 F}$ ).

369 Similarly, another study found that when adapted to a grass diet, Spodoptera littoralis had a smaller transcriptional response when fed maize compared to more generalist $S$. littoralis (Roy et 
371 al.2016). Determining the scale of these responsive regulatory effects and their role in toxin

372 resistance will help elucidate how D. sechellia has evolved to avoid the toxicity of M. citrifolia.

373 In response to OA, D. sechellia increases its expression of several Osiris genes (Lanno et

374 al. 2017). Previous work using RNAi in D. melanogaster to knock-down the expression of

375 individual genes and examine survival in these flies when exposed to OA showed that the

376 reduction of $O s i 6$, Osi7, and Osi8 expression decreased survival (Andrade Lopez et al. 2017). In

377 response to HA, neither $D$. sechellia nor D. melanogaster significantly alter the expression of

378 any Osiris genes, but D. simulans downregulates the expression of Osi6, Osi7, and Osi15 (Table

379 S2). The cellular and physiological function of these genes is unknown, so understanding what

380 these genes are doing and how they may be helping to shape the interactions between these

381 insects and their toxic hosts may be useful to understand how these interactions evolve (Coolon

382 et al.2019).

Examining and comparing the changes in transcriptional output of insects when exposed

384 to these different plant chemicals in specialized versus generalist species provides a framework

385 to understand how these interactions have evolved. Pathway analyses of these genes is useful in

386 determining the physiological function of altered expression in response to these chemicals, but

387 analyses of transcription factors that alter expression of these genes is necessary to better

388 understand the regulatory mechanisms involved in dietary specialization. Comparing

389 transcription factors responding to plant chemicals may help elucidate regulatory mechanisms

390 involved in these responses and shed light on how insects use changes in the transcription of

391 target genes in order to compete against plants in this evolutionary arms race to adapt to toxic

392 food sources. 
394 Table 1: Sequencing results and mapping percentage.

\begin{tabular}{cccccc}
\hline Sample & ID & \# of Reads & \# Mapped Reads & \% Mapped & Read length (nt) \\
\hline sim-C-1 & 105545 & 28056123 & 26210691 & $93.42 \%$ & 51 \\
sim-C-2 & 105546 & 26058213 & 24449785 & $93.82 \%$ & 51 \\
sim-C-3 & 105547 & 24095284 & 22589715 & $93.75 \%$ & 51 \\
sim-HA-1 & 105557 & 16844466 & 15844735 & $96.06 \%$ & 51 \\
sim-HA-2 & 105558 & 27002538 & 25521057 & $94.51 \%$ & 51 \\
sim-HA-3 & 105559 & 23222911 & 21786539 & $93.81 \%$ & 51 \\
mel-C-1 & 105542 & 21999530 & 20633866 & $93.79 \%$ & 51 \\
mel-C-2 & 105543 & 20950464 & 19779953 & $94.41 \%$ & 51 \\
mel-C-3 & 105544 & 22157160 & 20919514 & $94.41 \%$ & 51 \\
mel-HA-1 & 105554 & 19811200 & 18745553 & $94.62 \%$ & 51 \\
mel-HA-2 & 105555 & 23577339 & 22322344 & $94.68 \%$ & 51 \\
mel-HA-3 & 105556 & 18208025 & 17257250 & $94.78 \%$ & 51 \\
sec-C-1 & 76332 & 19222060 & 18496450 & $96.23 \%$ & 65 \\
sec-C-2 & 76333 & 20704811 & 19440620 & $93.89 \%$ & 65 \\
sec-C-3 & 76334 & 17696868 & 17123579 & $96.76 \%$ & 65 \\
sec-HA-1 & 76338 & 30612710 & 29079271 & $94.99 \%$ & 65 \\
sec-HA-2 & 76339 & 25873039 & 25202396 & $97.41 \%$ & 65 \\
sec-HA-3 & 76340 & 19074054 & 18031453 & $94.53 \%$ & 65 \\
\hline
\end{tabular}

395

\section{Figure Legends}

397 Figure 1: Experimental design and RNA-sequencing pipeline analysis. (A) 0-4 day old adult

398 female D. sechellia, D. simulans, and D. melanogaster flies were treated for 24 hours on either

399 control food or food supplemented with $0.23 \%$ hexanoic acid (HA). Flies were frozen in liquid

400 nitrogen, RNA was extracted, library prep was performed using poly-A selection, and libraries

401 were sequenced. (B) Raw sequencing reads were analyzed using FASTQC for quality control,

402 and then aligned to the respective reference genome using Bowtie2. Differential expression was

403 quantified using Cuffdiff using available respective genome annotation. Data was visualized

404 using R, and significantly differentially expressed genes (GRNs) were used for downstream

405 analysis. 
407 Figure 2: DEGs in adult D. melanogaster, D. simulans, and D. sechellia in response to HA. (AC) Plots showing DEG expression in control vs expression in HA treatment in (A) $D$.

409 melanogaster, (B) D. simulans, and (C) D. sechellia. Statistically significant genes are shown in 410 red. (D-F) Plots showing $\log _{2}$ (control/HA) fold change in (D) D. melanogaster, (E) D. simulans, 411 and (F) D. sechellia. Statistically significant genes are shown in red.

413 Figure 3: DEGs in D. melanogaster, D. simulans, and D. sechellia in response to HA. (A) The 414 number of differentially expressed genes in response to HA after RNA-seq are shown for each 415 species, D. sechellia, D. simulans and D. melanogaster. Overlap and species-specific number of 416 DEGs are indicated. (B) The number of differentially expressed genes in D. sechellia when it is 417 exposed to OA, HA, or L-DOPA treatment as well as the number of specific and overlapping 418 genes are shown.

420 Figure 4. (A) Significantly upregulated genes with D. melanogaster orthologs for each species 421 are shown by the percentage of genes of the total number upregulated genes that fall into each

422 KEGG pathway. (B) Significantly downregulated genes with D. melanogaster orthologs for each 423 species are shown by the percentage of genes out of the total number of downregulated genes 424 that fall into each KEGG pathway. The KEGG pathways Metabolic pathways and Toll and Imd 425 signaling were excluded. (C) Significantly downregulated genes with D. melanogaster orthologs 426 for each species are shown by the percentage of genes out of the total number of downregulated 427 genes that fall into the Metabolic pathways and Toll and Imd signaling pathway KEGG 428 pathways. 


\section{Acknowledgments}

430 Research reported in this publication was supported by Wesleyan University (Startup to JDC,

431 Department of Biology funds to JDC, College of the Environment funds to JDC), and the

432 National Institute Of General Medical Sciences of the National Institutes of Health under Award

433 Number R15GM135901 (award to JDC). The content is solely the responsibility of the authors

434 and does not necessarily represent the official views of the National Institutes of Health. 


\section{$\underline{\text { References }}$}

Afgan, E., Baker, D., van den Beek, M., Blankenberg, D., Bouvier, D., Čech, M., ... Goecks, J. (2016). The Galaxy platform for accessible, reproducible and collaborative biomedical analyses: 2016 update. Nucleic Acids Research, 44(W1), W3-W10. https://doi.org/10.1093/nar/gkw343

Altschul, S. F., Gish, W., Miller, W., Myers, E. W., \& Lipman, D. J. (1990). BLAST_article.pdf. Journal of Molecular Biology, Vol. 215, pp. 403-410. Retrieved from https://doi.org/10.1016/S0022-2836(05)80360-2

Alvarez-Curto, E., \& Milligan, G. (2016). Metabolism meets immunity: The role of free fatty acid receptors in the immune system. Biochemical Pharmacology, 114, 3-13. https://doi.org/10.1016/j.bcp.2016.03.017

Amlou, M., Moreteau, B., \& David, J. R. (1998). Genetic analysis of Drosophila sechellia specialization: Oviposition behavior toward the major aliphatic acids of its host plant. Behavior Genetics, 28(6), 455-464. https://doi.org/10.1023/A:1021689312582

Andrade Lopez, J. M., Lanno, S. M., Auerbach, J. M., Moskowitz, E. C., Sligar, L. A., Wittkopp, P. J., \& Coolon, J. D. (2017). Genetic basis of octanoic acid resistance in Drosophila sechellia : functional analysis of a fine-mapped region. Molecular Ecology, 1148-1160. https://doi.org/10.1111/mec.14001

Andrews, S. (2010). FastQC: a quality control tool for high throughput sequence data. Available at: Http://Www.Bioinformatics.Babraham.Ac.Uk/Projects/Fastqc.

Aranega-Bou, P., de la O Leyva, M., Finiti, I., Garcfa-Agustfn, P., \& Gonzalez-Bosch, C. (2014). Priming of plant resistance by natural compounds. Hexanoic acid as a model. Frontiers in Plant Science, 5(OCT), 1-12. https://doi.org/10.3389/fpls.2014.00488

Ashburner, M., Ball, C. A., Blake, J., Botstein, D., Butler, H., Cherry, J. M., ... Sherlock, G. (2000). Gene Ontology : tool for the. Gene Expression, 25(may), 25-29. https://doi.org/10.1038/75556

Auer, T. O., Khallaf, M. A., Silbering, A. F., Zappia, G., Ellis, K., Álvarez-Ocaña, R., ... Benton, R. (2020). Olfactory receptor and circuit evolution promote host specialization. Nature, 579(7799), 402-408. https://doi.org/10.1038/s41586-020-2073-7

Carbon, S., Douglass, E., Good, B. M., Unni, D. R., Harris, N. L., Mungall, C. J., Basu, S., Chisholm, R. L., Dodson, R. J., Hartline, E., Fey, P., Thomas, P. D., Albou, L. P., Ebert, D., Kesling, M. J., Mi, H., Muruganujan, A., Huang, X., Mushayahama, T., ... Elser, J. (2021). The Gene Ontology resource: Enriching a GOld mine. Nucleic Acids Research, 49(D1), D325-D334. https://doi.org/10.1093/nar/gkaa1113 
471

Chen, Y., \& Amrein, H. (2017). Ionotropic Receptors Mediate Drosophila Oviposition Preference through Sour Gustatory Receptor Neurons. Current Biology, 27(18), 27412750.e4. https://doi.org/10.1016/j.cub.2017.08.003

Coolon, J. D., Jones, K. L., Todd, T. C., Carr, B. C., \& Herman, M. A. (2009). Caenorhabditis elegans genomic response to soil bacteria predicts environment-specific genetic effects on life history traits. PLoS Genetics, 5(6). https://doi.org/10.1371/journal.pgen.1000503

Coolon, J. D., Webb, W., \& Wittkopp, P. J. (2013). Sex-specific effects of cis-regulatory variants in Drosophila melanogaster. Genetics, 195(4), 1419-1422. https://doi.org/10.1534/genetics.113.156331

Coolon, J. D., Drum, Z., Lanno, S. M., \& Smith, C. R. (2019). Osiris Genes and Insect Adaptive Evolution. ELS, (October). https://doi.org/10.1002/9780470015902.a0028763

Dushay, M. S., Åsling, B., \& Hultmark, D. (1996). Origins of immunity: Relish, a compound rellike gene in the antibacterial defense of Drosophila. Proceedings of the National Academy of Sciences of the United States of America, 93(19), 10343-10347. https://doi.org/10.1073/pnas.93.19.10343

Dworkin, I., \& Jones, C. D. (2009). Genetic changes accompanying the evolution of host specialization in Drosophila sechellia. Genetics, 181(2), 721-736. https://doi.org/10.1534/genetics.108.093419

Farine, J., Legal, L. U. C., Moreteauf, B., I, J. L. E. Q., Laboratoire, I., Recherches, D., Sully, R., De, L. M., Sud, U. P., Cedex, O., Populations, L., Yvette, G., Recherches, L. De, Bourgogne, U. De, De, L., \& F-, G. (1996). Volatile Components of Ripe Fruits of Morinda Citrifolia and their Effects on Drosophila. Phytochemistry, 4(2), 433-438. https://doi.org/10.1016/0031-9422(95)00455-6

García-Robles, I., Ochoa-Campuzano, C., Fernández-Crespo, E., Camañes, G., MartínezRamírez, A. C., González-Bosch, C., ... Real, M. D. (2013). Combining hexanoic acid plant priming with bacillus thuringiensis insecticidal activity against colorado potato beetle. International Journal of Molecular Sciences, 14(6), 12138-12156. https://doi.org/10.3390/ijms140612138

Groen, S. C., \& Whiteman, N. K. (2016). Using Drosophila to study the evolution of herbivory and diet specialization. Current Opinion in Insect Science, 14, 66-72. https://doi.org/10.1016/j.cois.2016.01.004

Hanson, M. A., \& Lemaitre, B. (2020). New insights on Drosophila antimicrobial peptide function in host defense and beyond. Current Opinion in Immunology, 62, 22-30. https://doi.org/10.1016/j.coi.2019.11.008 
Heidel-Fischer, H. M., \& Vogel, H. (2015). Molecular mechanisms of insect adaptation to plant secondary compounds. Current Opinion in Insect Science, 8, 8-14. https://doi.org/10.1016/j.cois.2015.02.004

Jaenike, J. (1990). Host specialization in phytophagous insects. Annual Review of Ecology and Systematics, 21(1), 243-273. https://doi.org/10.1146/annurev.es.21.110190.001331

Kanehisa, M., \& Sato, Y. (2020). KEGG Mapper for inferring cellular functions from protein sequences. Protein Science, 29(1), 28-35. https://doi.org/10.1002/pro.3711

Langmead, B., \& Salzberg, S. L. (2012). Fast gapped-read alignment with Bowtie 2. Nature Methods, 9(4), 357-359. https://doi.org/10.1038/nmeth.1923

Lavista-Llanos, S., Svatoš, A., Kai, M., Riemensperger, T., Birman, S., Stensmyr, M. C., \& Hansson, B. S. (2014). Dopamine drives Drosophila sechellia adaptation to its toxic host. ELife, 3, 1-17. https://doi.org/10.7554/eLife.03785

Lanno, S. M., Gregory, S. M., Shimshak, S. J., Alverson, M. K., Chiu, K., Feil, A. L., ... Coolon, J. D. (2017). Transcriptomic Analysis of Octanoic Acid Response in Drosophila sechellia Using RNA-Sequencing. G3\&amp;\#58; Genes|Genomes|Genetics, 7(December), g3.300297.2017. https://doi.org/10.1534/g3.117.300297

Lanno, S. M., Linde, S. C., Peyser, R. D., Shimshak, S. J., \& Coolon, J. D. (2019). Investigating the role of Osiris genes in Drosophila sechellia larval resistance to a host plant toxin. Ecology and Evolution, (December 2018), 1922-1933. https://doi.org/10.1002/ece3.4885

Lanno, S. M., \& Coolon, J. D. (2019). Derived esterase activity in Drosophila sechellia contributes to evolved octanoic acid resistance. Insect Molecular Biology, 28(6), 798-806. https://doi.org/10.1111/imb.12587

Legal, L., David, J. R., \& Jallon, J. M. (1992). Toxicity and attraction effects produced by Morinda citrifolia fruits on the Drosophila melanogaster complex of species. Chemoecology, 3, 125-129.

Legal, L., Chappe, B., \& Jallon, J. M. (1994). Molecular basis of Morinda citrifolia (L.): Toxicity on drosophila. Journal of Chemical Ecology, 20(8).

Login, F. H., Login, F. H., Balmand, S., Balmand, S., Vallier, A., Vallier, A., ... Heddi, A. (2011). Antimicrobial Peptides Keep Insect Endosymbionts Under Control. Science, 334(6054), 362-365. Retrieved from http://www.sciencemag.org/cgi/doi/10.1126/science.1209728\%5Cnpapers2://publication/do $\mathrm{i} / 10.1126 /$ science. 1209728

Masek, P., \& Keene, A. C. (2013). Drosophila Fatty Acid Taste Signals through the PLC Pathway in Sugar-Sensing Neurons. PLoS Genetics, 9(9). https://doi.org/10.1371/journal.pgen.1003710 
Matsuo, T., Sugaya, S., Yasukawa, J., Aigaki, T., \& Fuyama, Y. (2007). Odorant-binding proteins OBP57d and OBP57e affect taste perception and host-plant preference in Drosophila sechellia. PLoS Biology, 5(5), 0985-0996. https://doi.org/10.1371/journal.pbio.0050118

Mi, H., Muruganujan, A., Ebert, D., Huang, X., \& Thomas, P. D. (2019). PANTHER version 14: More genomes, a new PANTHER GO-slim and improvements in enrichment analysis tools. Nucleic Acids Research, 47(D1), D419-D426. https://doi.org/10.1093/nar/gky1038

Petschenka, G., \& Agrawal, A. A. (2016). How herbivores coopt plant defenses: Natural selection, specialization, and sequestration. Current Opinion in Insect Science, 14, 17-24. https://doi.org/10.1016/j.cois.2015.12.004

Peyser, R. D., Lanno, S. M., \& Shimshak, S. J. (2017). Analysis of cytochrome P450 contribution to evolved plant toxin resistance in Drosophila sechellia. 26, 715-720. https://doi.org/10.1111/imb.12329

R Core Development Team 2010 R: a language and environment for statis- tical computing (RDC Team, Ed). R Foundation for Statistical Computing, Vienna, Austria, ISBN:3900051-07-0.

R'Kha, S., Capy, P., \& David, J. R. (1991). Host-plant specialization in the Drosophila melanogaster species complex: A physiological, behavioral, and genetical analysis. Proceedings of the National Academy of Sciences of the United States of America, 88(5), 1835-1839. https://doi.org/10.1073/pnas.88.5.1835

Roy, A., Walker, W. B., Vogel, H., Chattington, S., Larsson, M. C., Anderson, P., ... Schlyter, F. (2016). Diet dependent metabolic responses in three generalist insect herbivores Spodoptera spp. Insect Biochemistry and Molecular Biology, 71, 91-105. https://doi.org/10.1016/j.ibmb.2016.02.006

Salazar-Jaramillo, L., Jalvingh, K. M., de Haan, A., Kraaijeveld, K., Buermans, H., \& Wertheim, B. (2017). Inter- and intra-species variation in genome-wide gene expression of Drosophila in response to parasitoid wasp attack. BMC Genomics, 18(1), 1-14. https://doi.org/10.1186/s12864-017-3697-3

Shokri, L., Inukai, S., Hafner, A., Weinand, K., Hens, K., Vedenko, A., ... Bulyk, M. L. (2019). A Comprehensive Drosophila melanogaster Transcription Factor Interactome. Cell Reports, 27(3), 955-970.e7. https://doi.org/10.1016/j.celrep.2019.03.071

Stork, N. E. (2018). How Many Species of Insects and Other Terrestrial Arthropods Are There on Earth? Annual Review of Entomology, 63(1), 31-45. https://doi.org/10.1146/annurevento-020117-043348 
Tauber, J. M., Brown, E. B., Li, Y., Yurgel, M. E., Masek, P., \& Keene, A. C. (2017). A subset of sweet-sensing neurons identified by IR56d are necessary and sufficient for fatty acid taste. PLoS Genetics, 13(11), 1-18. https://doi.org/10.1371/journal.pgen.1007059

Thurmond, J., Goodman, J. L., Strelets, V. B., Attrill, H., Gramates, L. S., Marygold, S. J., ... Baker, P. (2019). FlyBase 2.0: The next generation. Nucleic Acids Research, 47(D1), D759-D765. https://doi.org/10.1093/nar/gky1003

Trapnell, C., Williams, B. A., Pertea, G., Mortazavi, A., Kwan, G., Van Baren, M. J., ... Pachter, L. (2010). Transcript assembly and quantification by RNA-Seq reveals unannotated transcripts and isoform switching during cell differentiation. Nature Biotechnology, 28(5), 511-515. https://doi.org/10.1038/nbt.1621

Ungerer, M. C., Johnson, L. C., \& Herman, M. A. (2008). Ecological genomics: Understanding gene and genome function in the natural environment. Heredity, 100(2), 178-183. https://doi.org/10.1038/sj.hdy.6800992

Vilcinskas, A. (2013). Evolutionary plasticity of insect immunity. Journal of Insect Physiology, 59(2), 123-129. https://doi.org/10.1016/j.jinsphys.2012.08.018

Watanabe, K., Kanaoka, Y., Mizutani, S., Uchiyama, H., Yajima, S., Watada, M., ... Hattori, Y. (2019). Interspecies Comparative Analyses Reveal Distinct Carbohydrate-Responsive Systems among Drosophila Species. Cell Reports, 28(10), 2594-2607.e7. https://doi.org/10.1016/j.celrep.2019.08.030 


\section{Experimental Design}

$\begin{array}{lll}\text { D. melanogaster } & \text { D. simulans } & \text { D. sechellia }\end{array}$

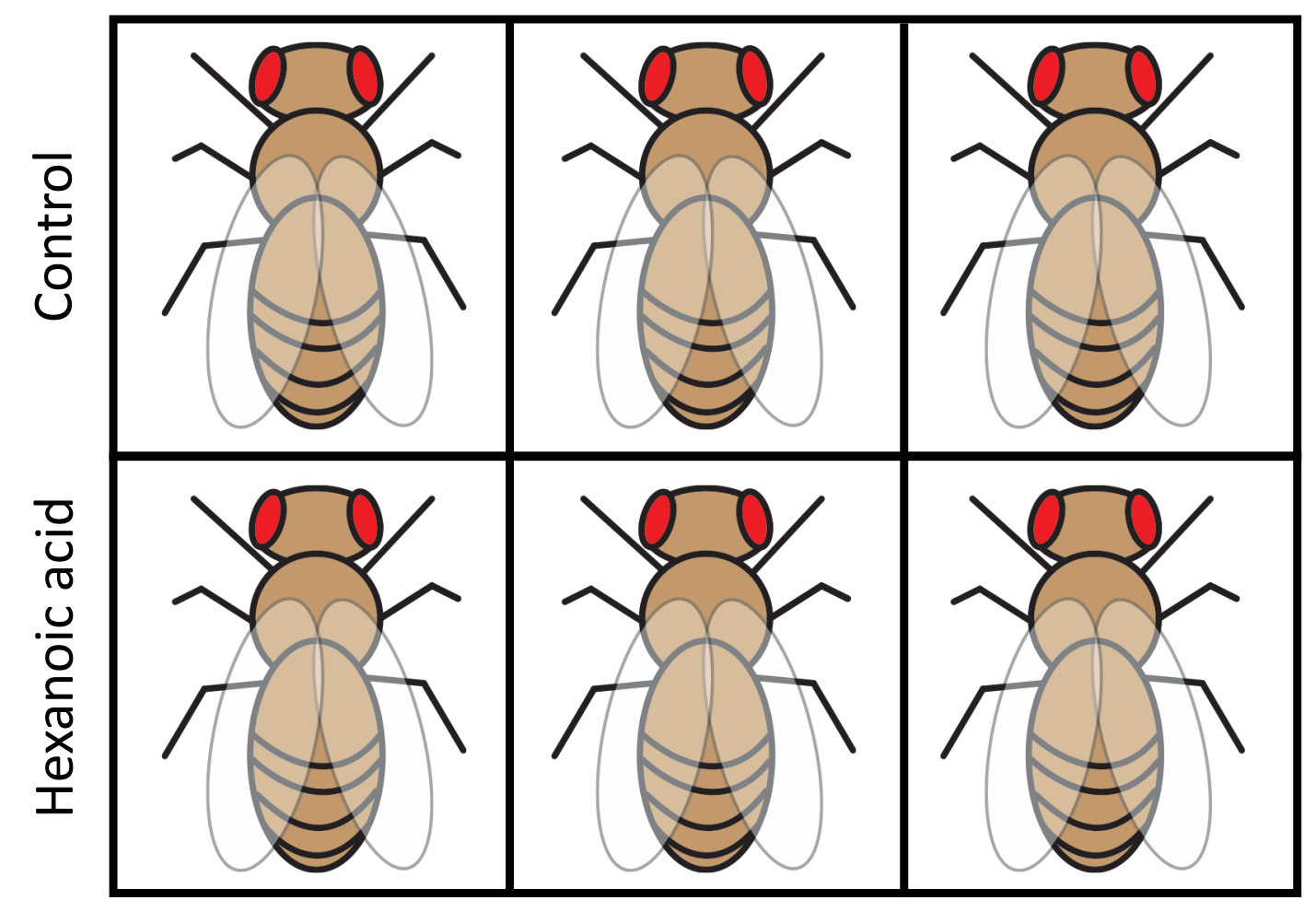

Collect samples Extract RNA Library prep Nextseq

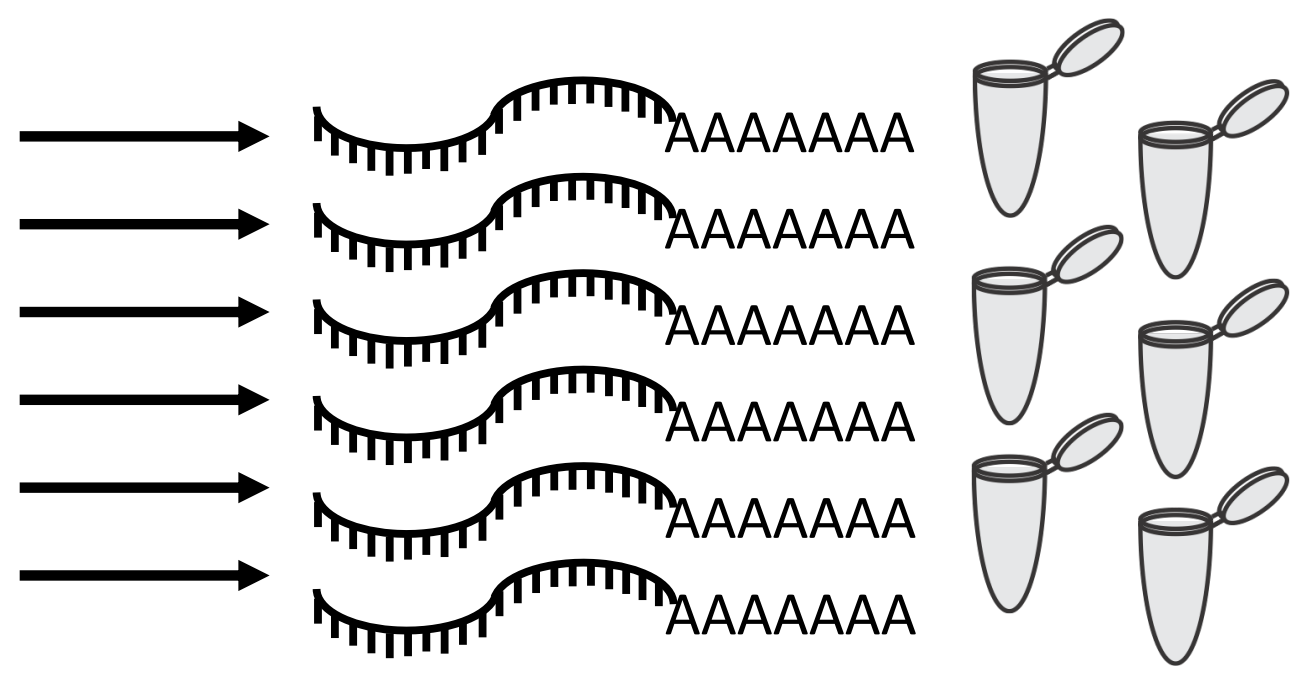

eseq 1 +seq 1

ATGATGATGATGATGA

FGGGHHFGHFGHFGFH

Data Interpretation

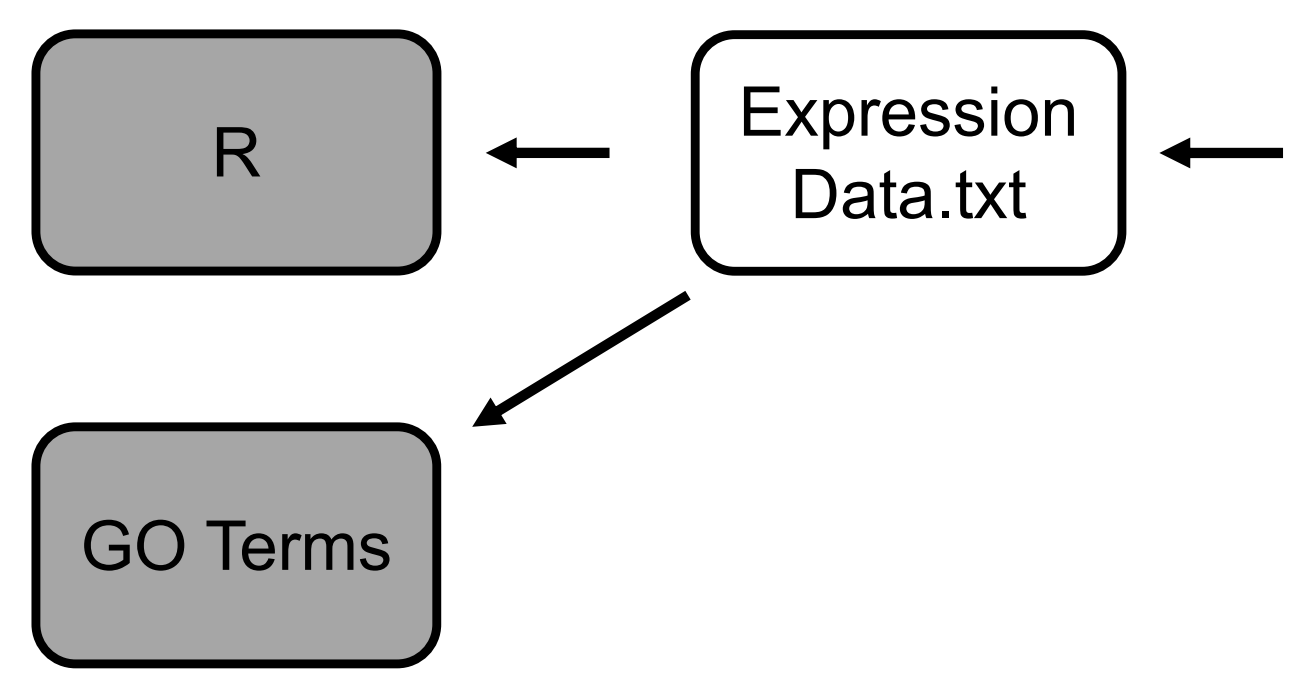

Statistical Analysis

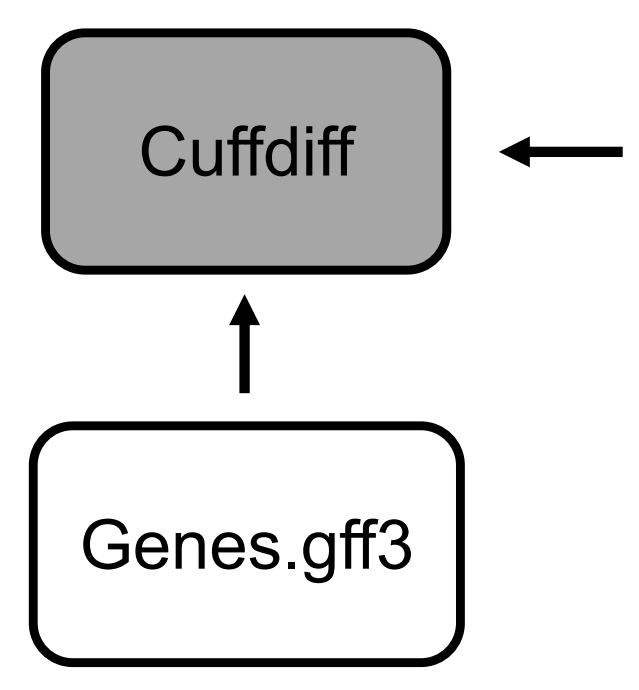

Sequence Alignment

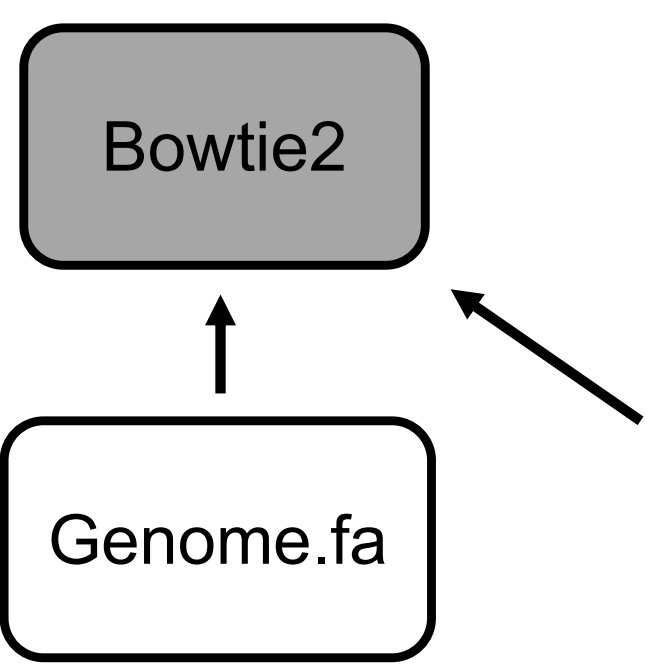

Quality Control

FASTQC

Reads.fastq 

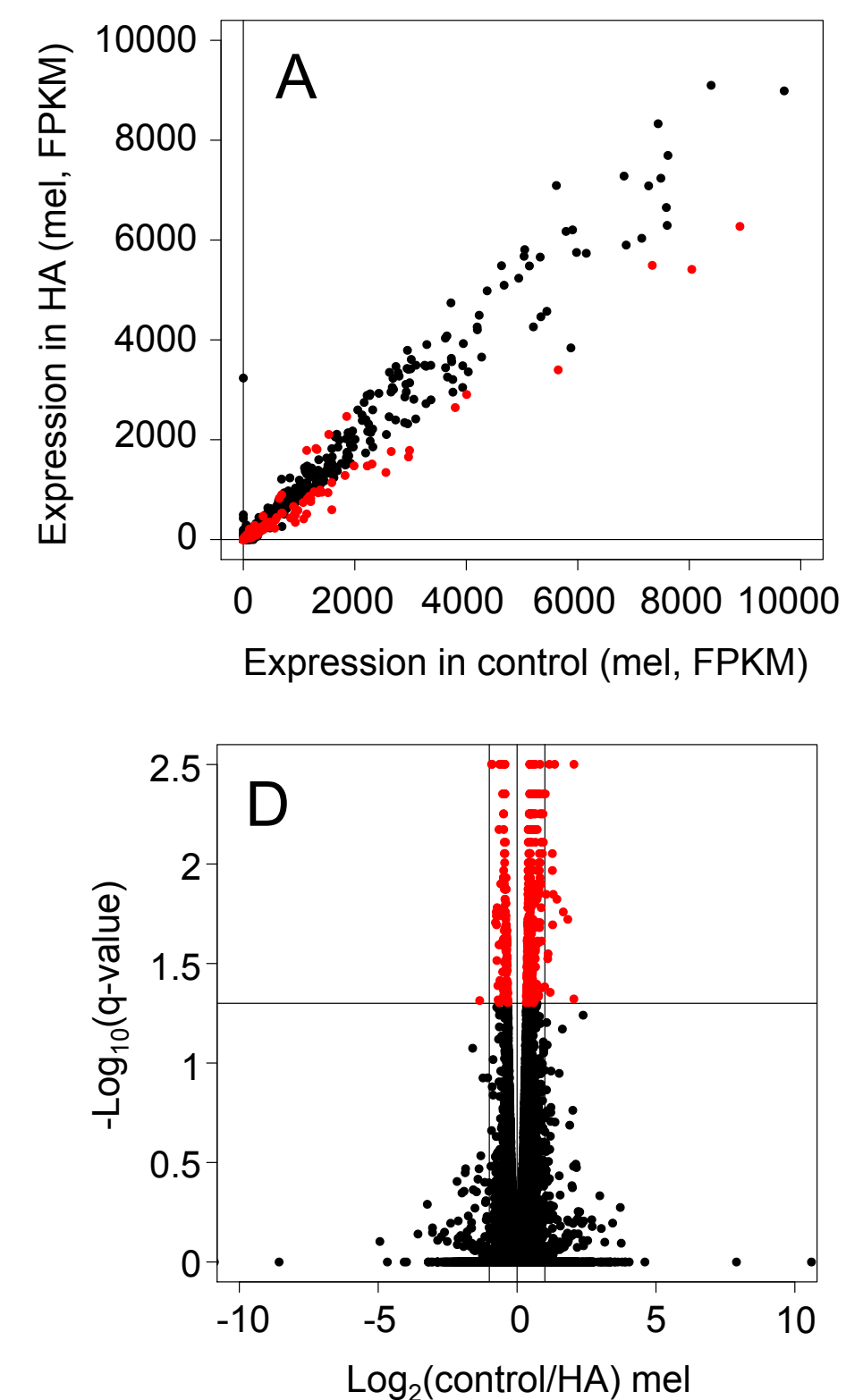
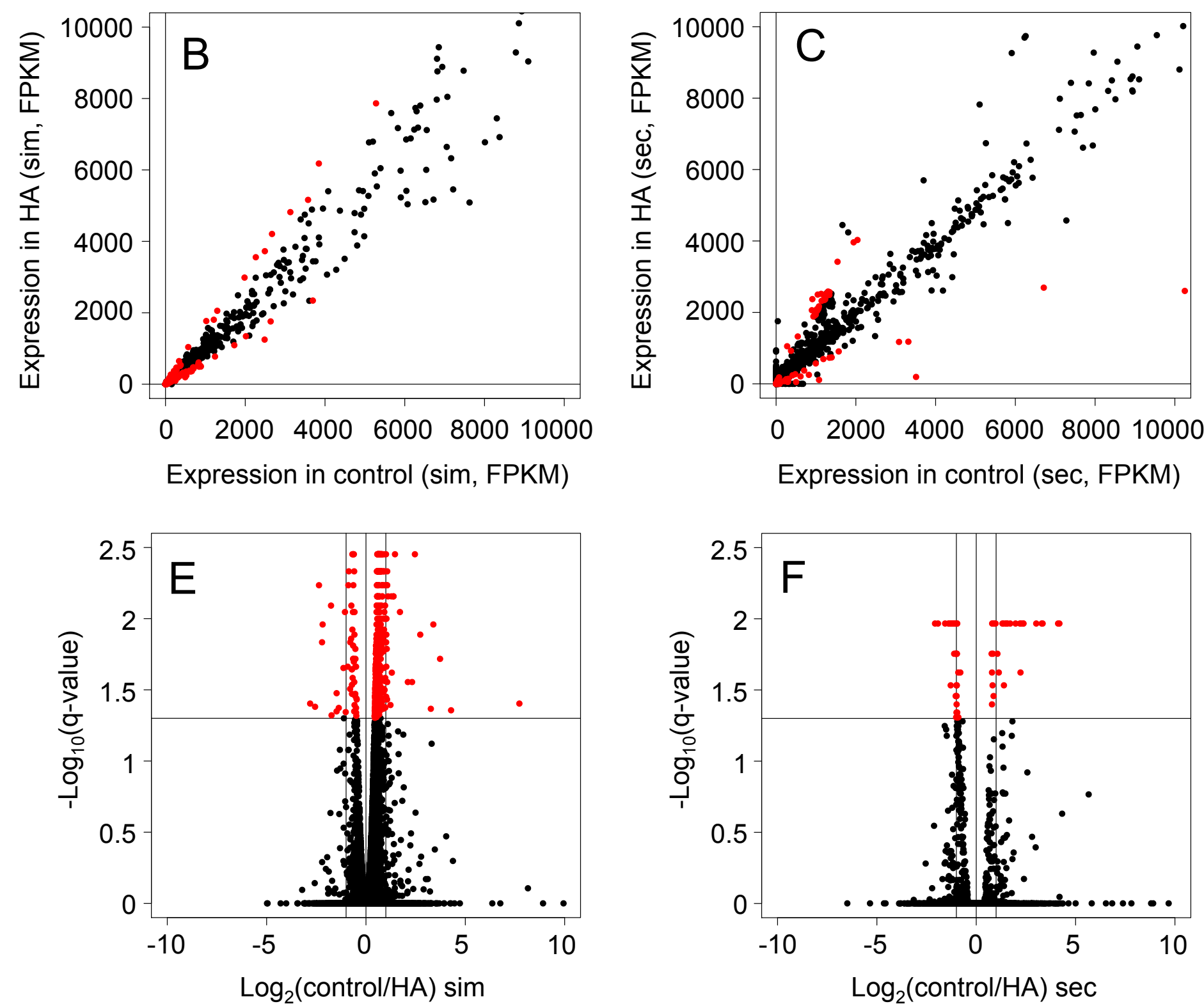

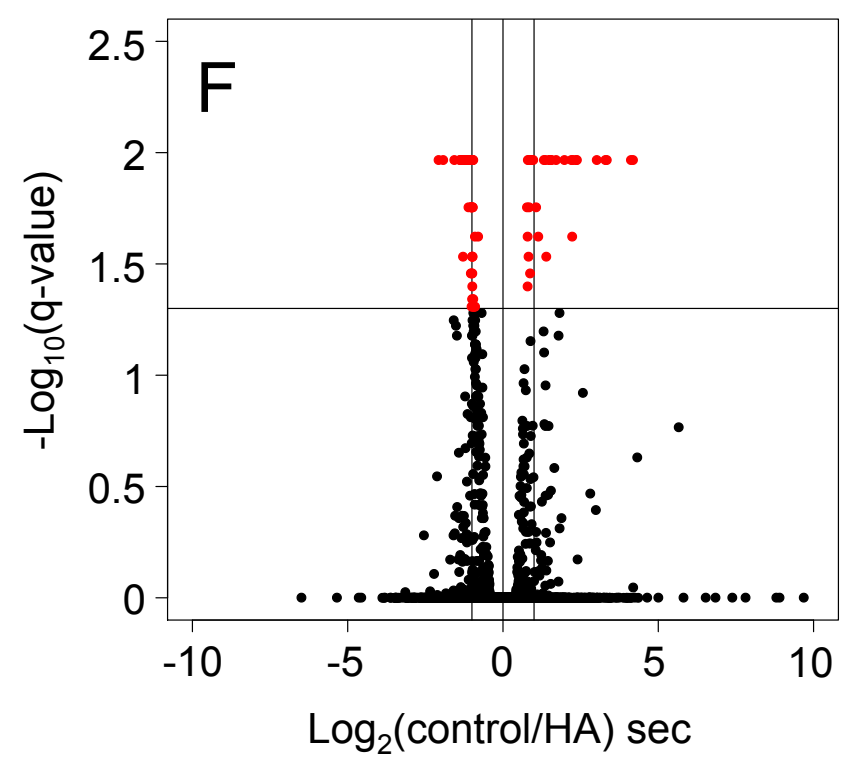




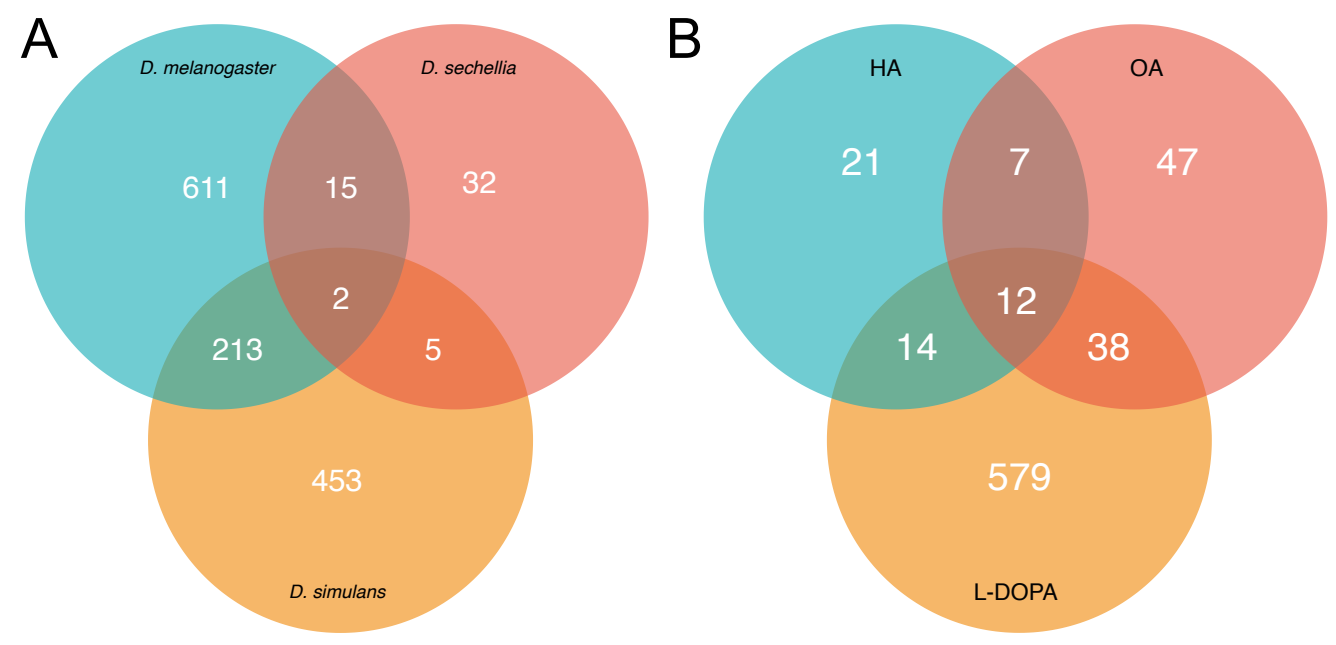




\section{A}

Aminoacyl-tRNA biosynthesis

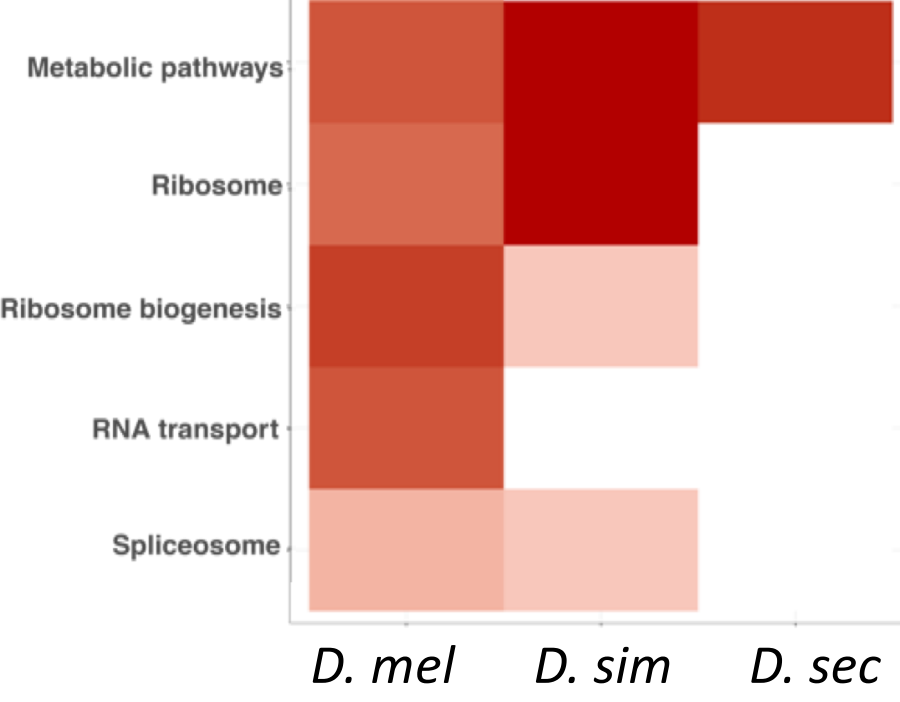

C

Metabolic pathways

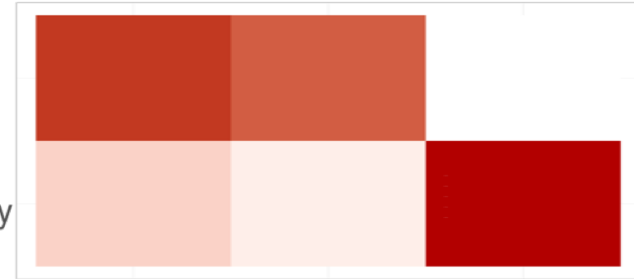

D. mel

D. sim

D. sec

\section{$\%$ upregulated genes}

6

4

2

0

$\%$ downregulated genes

\section{0}

5

0
B
AutophagyBiosynthesis of unsaturated fatty acids-
Carbon metabolismDrug metabolism - cytochrome P450 Drug metabolism - other enzymes Fatty acid degradation Fatty acid metabolism Galactose metabolism Glutathione metabolism Glycerolipid metabolism Hedgehog signaling pathway MAPK signaling pathway Metabolism of xenobiotics by cytochrome P450 mTOR signaling pathway Neuroactive ligand-receptor interaction
Oxidative phosphorylation
Pentose and glucuronate interconversions
Peroxisome Neuroactive ligand-receptor interaction
Oxidative phosphorylation
Pentose and glucuronate interconversions Pentose and glucuronate Porphyrin and chlorophyll metabolism Protein expor Protein processing in ER
Pyrimidine metabolism Ribosome biogenesis RNA polymerase RNA transport Spliceosome Starch and sucrose metabolism Tyrosine metabolism Wnt signaling pathway Pyrimidine metabolism
$\%$ downregulated genes 


\section{OA and HA KEGG Pathways}

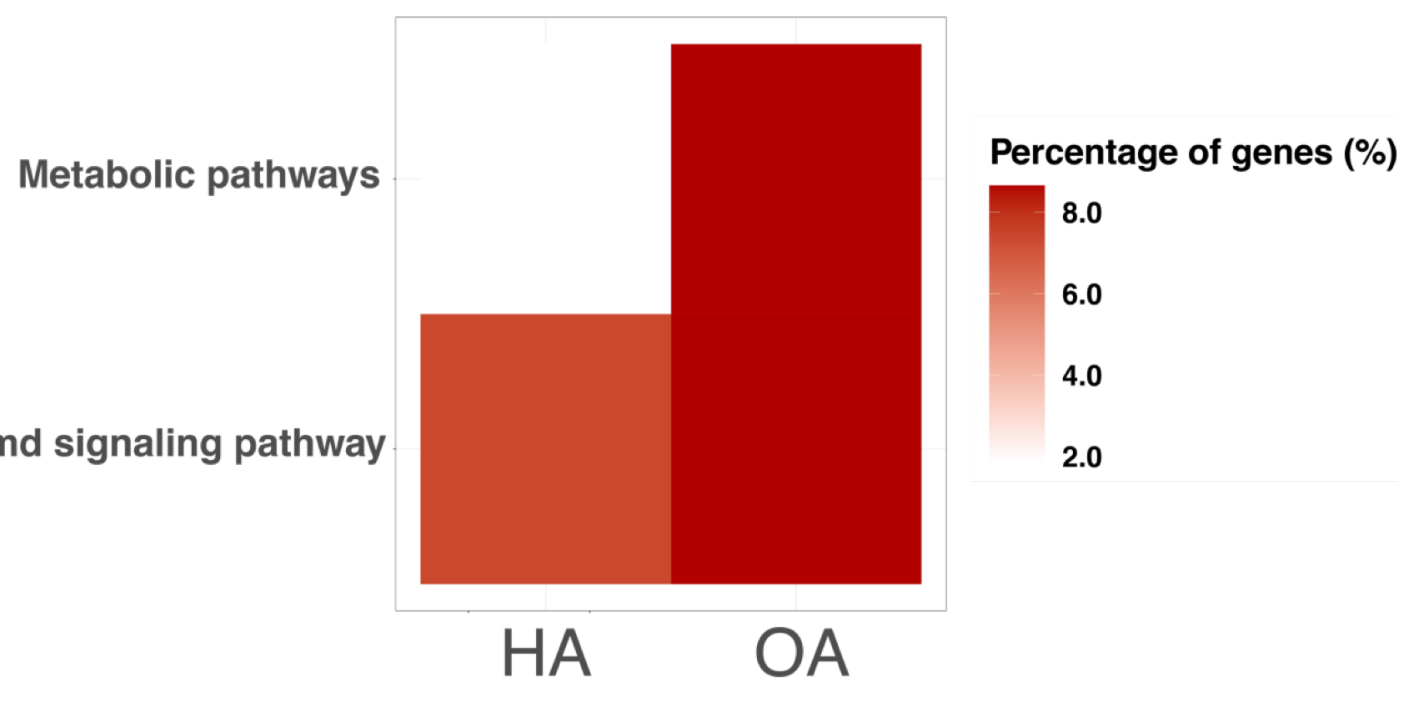

